\title{
A CASE OF TUBERCULOUS PERICARDIAL EFFUSION
}

Tuberculosis accounts for up to $4 \%$ of acute pericarditis and $7 \%$ cases of cardiac tamponade. Prompt treatment can be life saving but requires accurate diagnosis. We report a case of 30 -year-old male who presented with fever, chills, and dry nonproductive cough since one month. The case was diagnosed by radiological findings, which were suggestive of pulmonary tuberculosis, followed by acid fast staining and culture of the aspirated pericardial fluid. The patient was responding to antitubercular treatment at the last follow up.

Key words: Adult, tuberculous pericarditis

Although there has been a significant decline in tuberculosis in wealthy industrialized countries over last decades, Africa, Asia, and Latin America with $86 \%$ of the world's population, are home to $95 \%$ of all cases of active tuberculosis and $98 \%$ of nearly two million deaths resulting from tuberculosis each year. ${ }^{[1]}$ Tuberculous pericarditis, caused by Mycobacterium tuberculosis is found approximately in $1 \%$ of all autopsied cases of tuberculosis and in $1-2 \%$ instances of pulmonary tuberculosis. ${ }^{[2]}$ Prompt treatment of tuberculous pericarditis can be life saving. Effective treatment requires a rapid and accurate diagnosis but is often difficult. ${ }^{[3]}$ Here, we report a case of tuberculous pericarditis in a 30-year-old male, diagnosed by acid fast stain examination on the aspirated fluid who was treated promptly with antitubercular drugs.

\section{Case Report}

A 30-year-old male patient presented on the $6^{\text {th }}$ December 2006, with fever associated with chills and a dry, nonproductive cough since one month. There was no history of tuberculosis, diabetes, or immunosuppression.

On examination, he was febrile, pulse rate of $86 / \mathrm{min}$, blood pressure of 110/70 $\mathrm{mm} \mathrm{Hg}$, and respiratory rate of 16/ min. Jugular venous pulse was raised and on auscultation, the heart sounds were muffled and associated with a pericardial rub. There was no peripheral edema, cyanosis, pallor, icterus or hepatosplenomegaly. 
Laboratory investigations revealed $\mathrm{Hb}$ of $14 \mathrm{~g} / \mathrm{dl} ; \mathrm{RBC}$ count of 4.72 million $/ \mathrm{mm}^{3}$; WBC count of $17,000 / \mathrm{mm}^{3}$ with polymorphs $60 \%$, lymphocytes $37 \%$, eosinophils $2 \%$ and monocytes $1 \%$, platelet counts of $3.14 \mathrm{lac} / \mathrm{mm}^{3}$, and an ESR of $40 \mathrm{~mm}$. He was seronegative for HIV and the hepatic and renal function tests were within normal limits. ECG showed low voltage complexes with sinus tachycardia. Chest X-ray indicated left basal consolidation with effusion along with cardiomegaly (Fig. 1). An ultrasound guided pigtail catheter was inserted. Over the next few days $1000 \mathrm{ml}$ of strawcolored pericardial fluid was drained.

The drained fluid was sent for microbiological examination. The Ziehl-Neelsen (ZN) stained smears showed acid fast bacilli (AFB) along with pus cells Fig. 2. Culture on Lowenstein Jensen (LJ) media showed rough and buff colonies suggestive of $M$. tuberculosis Fig. 3 after four weeks of incubation and was confirmed by acid fast staining (with 25\% sulfuric acid as decolorizer), and biochemical tests. Three early morning sputum samples were examined

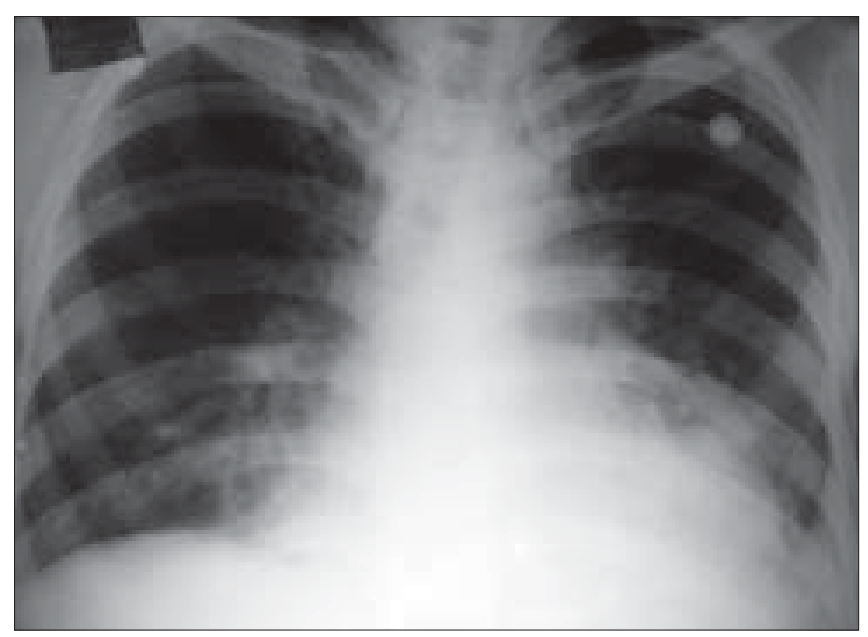

Figure 1: Chest X-ray indicating left basal consolidation with effusion along with cardiomegaly and diffuse interstitial pulmonary infiltrations notably in the right basal lobe of the lungs

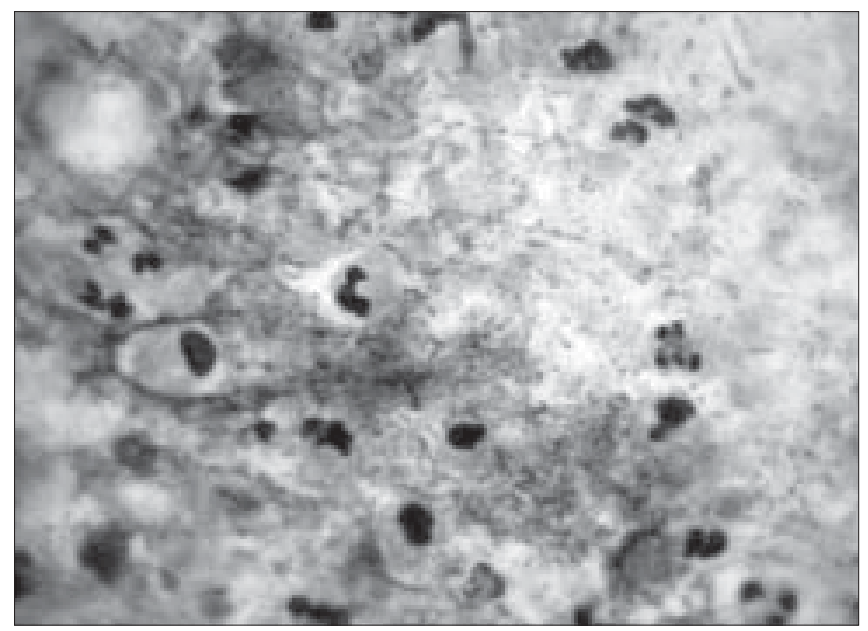

Figure 2: $\mathrm{ZN}$ stained smear of pericardial fluid showing AFB in pus cells by $\mathrm{ZN}$ stain and LJ culture but were negative for AFB and the culture showed no growth after eight weeks of incubation.

The patient responded well to the pericardial fluid drainage with subsidence of the dyspnea. He was started on isoniazid $300 \mathrm{mg}$, rifampicin $450 \mathrm{mg}$, pyrazinamide $1250 \mathrm{mg}$, and ethambutol $750 \mathrm{mg}$. The fever subsided over a period of two weeks and the patient was discharged one month from the date of admission, on the above medications. The patient was responding well to the treatment, with no recurrence of symptoms or any signs of deterioration when last followed up, eight weeks after the start of therapy.

\section{Discussion}

Extrapulmonary tuberculosis occurs in $20 \%$ of patients with tuberculosis. Tuberculous pericarditis is seen in $1-8 \%$ of these patients. ${ }^{[4]}$ Tuberculous pericarditis is the most common cause of pericarditis in Africa and other countries where tuberculosis remains a major problem. ${ }^{[4]}$ The incidence of tuberculous pericarditis is increasing with the advent of the AIDS pandemic. Tuberculosis accounts for up to $4 \%$ of acute pericarditis and $7 \%$ of cardiac tamponade. The mortality rate of tuberculosis still ranges from $14-40 \%$. Tuberculous pericarditis is a potentially lethal condition. The pericardial effusion is mainly due to hypersensitivity to tubercular protein. ${ }^{[5]}$

The route of spread of the organisms to pericardium is usually from mediastinal or hilar lymph nodes or from lungs or rarely as a part of miliary tuberculosis. Typically, the process begins as effusive constrictive pericarditis. In later stages, AFB are usually not detected but caseating granulomas involving the pericardium and epicardium may be present. ${ }^{[6]}$

Tuberculous pericarditis has a variable clinical presentation and should be considered in the evaluation

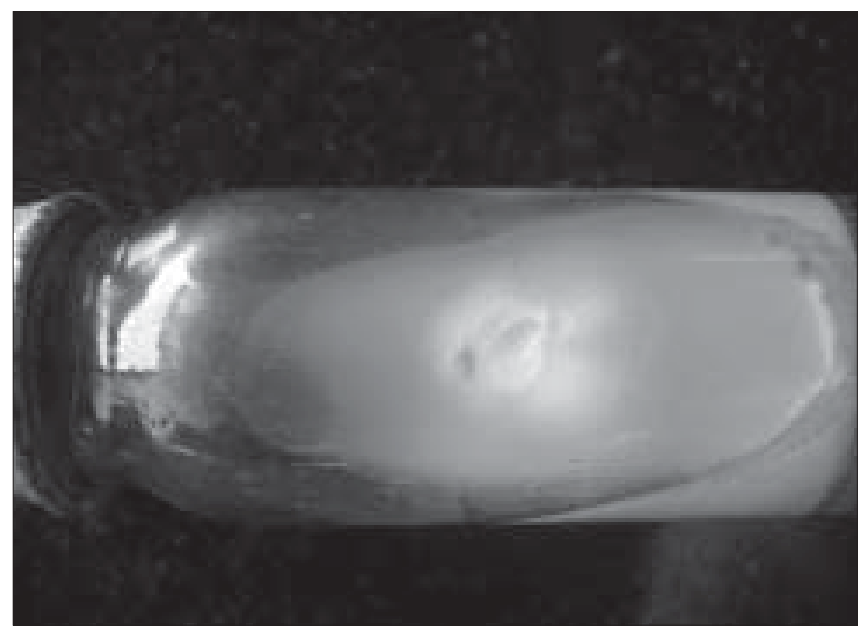

Figure 3: LJ medium showing rough and buff colored colonies of $M$. tuberculosis observed after four weeks of incubation 
of all cases of pericarditis without a rapidly self-limiting course. ${ }^{[6]}$ Tuberculous pericardial effusion develops insidiously, presenting with symptoms such as fever, night sweats, fatigue, weight loss, chest pain, cough, breathlessness, although severe pericardial pain of acute onset of idiopathic pericarditis is unusual. In some cases, evidence of chronic cardiac compression mimicking heart failure is common presentation. ${ }^{[6]}$ Cardiac tamponade may present as a complication of pericardial effusion. ${ }^{[6]}$ In the present case, any such complications were averted due to the early diagnosis and prompt treatment. Prompt treatment of tuberculous pericarditis may be life saving. ${ }^{[3]}$ Effective treatment requires a rapid and accurate diagnosis, which is often difficult. ${ }^{[3]}$

Chest radiograph which shows an enlarged cardiac shadow in more than $90 \%$ cases, demonstrates features of active pulmonary disease in $30 \%$ cases. $^{[4,6]}$ The ECG is abnormal in virtually all cases of tuberculous pericarditis ${ }^{[6,7]}$ as observed in the present case.

Definite tuberculous pericarditis can be diagnosed by one or more of the following criteria: ${ }^{[6]}$

- Isolation of M. tuberculosis from pericardial effusion fluid or pericardial biopsy.

- Demonstration of granulomatous inflammation on histologic examination of pericardial biopsy sample.

- Isolation of $M$. tuberculosis from sputum or non pericardial effusion exudates in the presence of clinical and/or radiological evidence of tuberculosis, associated with a positive response to antitubercular therapy and in the absence of any other obvious cause for pericarditis.

In the present case, the diagnosis was confirmed by demonstration of AFB in aspirated material and isolation on LJ medium.

The variability in the detection of tubercle bacilli in a direct smear of pericardial fluid is well documented but the yield may range from $0-42 \% \cdot{ }^{[8]}$ Hence, culture is said to be the gold standard.

Rapid diagnosis by polymerase chain reaction (PCR) was considered an important diagnostic tool, till a study by
Cegieiski et al ${ }^{\left[{ }^{[9]}\right.}$ demonstrated that the sensitivity of PCR of pericardial tissue is comparatively lower than culture.

Antibiotic chemotherapy increases survival dramatically in tuberculous pericarditis. A regimen consisting of isoniazid, rifampicin, pyrazinamide, and ethambutol for at least two months followed by INH and rifampicin (total six months of therapy) have been shown to be highly effective. ${ }^{[3,6]}$

\section{References}

1. Murray JF. A century of tuberculosis. Am J Respir Crit Care Med 2004;169:1181-6.

2. Fowler NO. Tuberculous pericarditis. JAMA 1991;266:99103.

3. Reuter H, Burgess L, van Vuuren W, Donbell A. Diagnosing tuberculous pericarditis. Q J Med 2006;99:827-39.

4. Lorell BH, Braunwald E. In: Heart disease. $4^{\text {th }}$ ed. Philadelphia: Saunders WB; 1992. p. 1456-507.

5. Agrawal S, Radhakrishnan S, Sinha N. Echocardiographic demonstration of resolving intrapericardial mass in tuberculous pericardial disease. Int J Cardiol 1990;26:240-1.

6. Mayosi BM, Burgess LJ, Doubell AF. Tuberculous pericarditis: Heart disease in Africa. Circulation 2005;112:3608-16.

7. Smedmea JP, Katjitae I, Reuter H, Burgess L, Louw V, Pretorius M, et al. Twelve lead electrocardiography in tuberculous pericarditis. Cardiovasc J Surg Afr 2001;12:31-4.

8. Sagrista-Sauleda J, Permanyer-Miralda G, Soler-Soler J. Tuberculous pericarditis: Ten year experience with prospective protocol for diagnosis and treatment. J Am Coll Cardiol 1988;11:724-8.

9. Cegielski JP, Delvin BH, Morris AJ, Kitinya JN, Pulipaka UP, Lema LE, et al. Comparison of PCR, culture and histopathology for diagnosis of tuberculous pericarditis. J Clin Microbiol 1997;35:3254-7.

K Wanjari, *V Baradkar, M Mathur, S Kumar

Department of Microbiology, Lokmanya Tilak Municipal Medical College General Hospital, Sion, Mumbai - 400 022, India

*Corresponding author (email: <vasantbaradkar@yahoo.com>)

Submission : 9-07-2008

Accepted : 13-08-2008 